\title{
COUNTABLE PARACOMPACTNESS OF $\Sigma$-PRODUCTS
}

\author{
LECHENG YANG \\ (Communicated by Franklin D. Tall)
}

\begin{abstract}
It is known that $\Sigma$-products of compact spaces always are countably paracompact but not necessarily normal. In the present paper it is proved that a $\Sigma$-product of paracompact $\sigma$-spaces is normal if and only if it is countably paracompact.
\end{abstract}

\section{INTRODUCTION}

The equivalence of normality and countable paracompactness in Cartesian products has been investigated by many authors $[5,8,9,17,22]$ so that this topic constitutes a very interesting part in the theory of product spaces [7, 14]. In this paper the equivalence of normality and countable paracompactness will be considered for $\Sigma$-products.

The concept of $\Sigma$-products was introduced by Corson [2] who proved that $\Sigma$-products of complete metric spaces are normal. In Gul'ko [4] and Rudin [15] the following is shown:

(i) A $\Sigma$-product of metric spaces is normal.

This answers affirmatively a long outstanding question raised by Corson [2]. Kombarov [8] later generalized (i) by obtaining the following result:

(ii) A $\Sigma$-product of paracompact $p$-spaces is (collectionwise) normal if and only if it has countable tightness.

In connection with the above results, the following Questions 1 and 2 are considered by Yajima [18] and Kodama, respectively.

Question 1. Is a $\Sigma$-product of paracompact $\sigma$-spaces normal if it has countable tightness?

Question 2. Is a $\Sigma$-product of Lašnev spaces normal?

Question 1 has been answered positively. In fact Yajima [18] even proved

(iii) A $\Sigma$-product of paracompact $\Sigma$-spaces is (collectionwise) normal if it has countable tightness.

Since paracompact $p$-spaces are $\Sigma$-spaces, (iii) is also a generalization of the "if" part of (ii). However, the countable tightness is no longer a necessary condition for a $\Sigma$-product of paracompact $\Sigma$-spaces to be normal, because there

Received by the editors March 3, 1993.

1991 Mathematics Subject Classification. Primary 54B10, 54D10, 54D18.

Key words and phrases. $\Sigma$-product, $\sigma$-space, countably paracompact, normal. 
exists a collectionwise normal $\Sigma$-product of $M_{1}$-spaces which has no countable tightness [18]. Moreover, since there exists a nonnormal $\Sigma$-product of $M_{1}$ spaces [18], in Question 1 the assumption of countable tightness cannot be dropped. On the other hand, Rudin [16] proved that any $\Sigma$-product of metric spaces is shrinking and hence countably paracompact. It is also known from Yajima [19] that any normal $\Sigma$-product of $\sigma$-spaces is countably paracompact.

The main purpose of this paper is to establish the equivalence of normality and countable paracompactness of $\Sigma$-products of paracompact $\sigma$-spaces. Namely we prove the following theorem.

Theorem 1. $A \Sigma$-product of paracompact $\sigma$-spaces is normal if and only if it is countably paracompact.

In the rest of the paper, we also consider the subshrinking property of $X \times \kappa$, where $X$ is a semistratifiable space and $\kappa$ an uncountable regular cardinal with the usual order topology. The subshrinking property was introduced by Yajima [20] which is important for the study of shrinking property (see Yajima [20] and Hoshina [6]). Yajima [21] recently proved that $X \times \kappa$ is subshrinking for any $\sigma$-space $X$, and he asked whether $X \times \kappa$ is subshrinking for a semistratifiable space $X$. We shall prove

Theorem 2. Let $X$ be a semistratifiable space with $\chi(X)<\kappa$. Then $X \times \kappa$ is subshrinking.

All spaces considered here are assumed to be regular $T_{1}$. The set of natural numbers is denoted by $\mathbb{N}$ and natural numbers are denoted by $i, j, k$, and $n$. $\kappa$ always denotes an uncountable regular cardinal with the usual order topology.

\section{Proof of Theorem 1}

Let $X=\prod_{\lambda \in \Lambda} X_{\lambda}$ be the Cartesian product of spaces $X_{\lambda}, \lambda \in \Lambda$, and let $s=\left(s_{\lambda}\right)_{\lambda \in \Lambda}$ be a fixed point of $X$. The subspace $\Sigma=\left\{x \in X: x_{\lambda}=s_{\lambda}\right.$ for all but countably many $\lambda \in \Lambda\}$ of $X$ is called a $\Sigma$-product of spaces $X_{\lambda}, \lambda \in \Lambda$. Such a point $s \in \Sigma$ is called the base point of $\Sigma$, which is often omitted.

Let $X$ be a $\Sigma$-product of spaces $X_{\lambda}, \lambda \in \Lambda$, with a base point $\left(s_{\lambda}\right)_{\lambda \in \Lambda}$. For a point $x \in X$, denote by $\operatorname{Supp}(x)$ the set $\left\{\lambda \in \Lambda: x_{\lambda} \neq s_{\lambda}\right\}$. Let $\Delta$ be an index set such that for each $\xi \in \Delta, R_{\xi}$ is a subset of $\Lambda$. Then we denote by $X_{\xi}$ the Cartesian product $\prod_{\lambda \in R_{\xi}} X_{\lambda}$ and by $p_{\xi}$ the projection of $X$ onto $X_{\xi}$ for each $\xi \in \Delta$.

Let $\xi=\left(\alpha_{i j}\right)_{i, j \leq n}$ be an $n \times n$ matrix. By $\xi_{k}$ we denote the $k \times k$ matrix $\left(\alpha_{i j}\right)_{i, j \leq k}$ for $1 \leq k \leq n$. In particular, $\xi_{n-1}$ is often abbreviated as $\xi_{-}$and $\xi_{0}$ denotes the empty set $\varnothing$.

A space is called a $\sigma$-space if it has a $\sigma$-locally finite net [13]. Note that Lašnev spaces (i.e., closed images of metric spaces) are $M_{1}$, and $M_{1}$-spaces are paracompact $\sigma$ [13]. It is well known that the countable product of paracompact $\sigma$-spaces ( $\Sigma$-spaces) is paracompact $\sigma(\Sigma)$.

The following two lemmas are useful to prove Theorem 1.

Lemma 1 [5, Lemma 2.1]. Let $X$ be a countably paracompact space and let $E$ and $F$ be a pair of disjoint subsets. Suppose that $F$ is closed and there exists open sets $U_{n}, n \in \mathbb{N}$, such that $E \subset \bigcap_{n \in \mathbb{N}} U_{n}$ and $\left(\bigcap_{n \in \mathbb{N}} \bar{U}_{n}\right) \cap F=\varnothing$. Then $E$ and $F$ are separated by open sets. 
Lemma 2 [11, Theorem 1]. Let $X$ be a $\sigma$-space. Then there exists a sequence $\left\{\mathscr{F}_{n}: n \in \mathbb{N}\right\}$ of locally finite closed covers of $X$, satisfying

(a) $\mathscr{F}_{n}=\left\{F\left(\alpha_{1} \cdots \alpha_{n}\right): \alpha_{1}, \ldots, \alpha_{n} \in \Omega\right\}$ for each $n \in \mathbb{N}$,

(b) $F\left(\alpha_{1} \cdots \alpha_{n}\right)=\bigcup\left\{F\left(\alpha_{1} \cdots \alpha_{n} \alpha\right): \alpha \in \Omega\right\}$ for each $\alpha_{1}, \ldots, \alpha_{n} \in \Omega$,

(c) For each $x \in X$, there exists a sequence $\alpha_{1}, \alpha_{2}, \ldots \in \Omega$ such that $x \in$ $\bigcap_{n \in \mathbb{N}} F\left(\alpha_{1} \cdots \alpha_{n}\right)$ and each open $n b d$ of $x$ contains some $F\left(\alpha_{1} \cdots \alpha_{n}\right)$.

The above sequence $\left\{\mathscr{F}_{n}: n \in \mathbb{N}\right\}$ is called a spectral $\sigma$-net of $X$ and the sequence $\left\{F\left(\alpha_{1} \cdots \alpha_{n}\right): n \in \mathbb{N}\right\}$ in (c) is called a local $\sigma$-net of $X$ at $x$.

Our proof of Theorem 1 is based on the idea in Yajima [18, 20] and we shall use the following fact: a space $X$ is normal if and only if for every pair $A, B$ of disjoint closed subsets of $X$ there exists a $\sigma$-locally finite open cover $\mathscr{U}$ of $X$ such that either $\bar{U} \cap A=\varnothing$ or $\bar{U} \cap B=\varnothing$ for every $U \in \mathscr{U}$.

Proof of Theorem 1. Let $X$ be a $\Sigma$-product of paracompact $\sigma$-spaces $X_{\lambda}$, $\lambda \in \Lambda$, with a base point $s=\left(s_{\lambda}\right)_{\lambda \in \Lambda} \in X$, and suppose $X$ is countably paracompact. To prove that $X$ is normal, let $A$ and $B$ be a pair of disjoint closed subsets of $X$; we shall find a $\sigma$-locally finite open cover $\mathscr{G}$ of $X$ such that either $\bar{U} \cap A=\varnothing$ or $\bar{U} \cap B=\varnothing$ for every $U \in \mathscr{U}$. Let $\Delta_{0}=\left\{\xi_{0}\right\}$, where $\xi_{0}=(\varnothing)$, and take an arbitrary nonempty countable subset $R_{\xi_{0}} \subset \Lambda$.

Now, for each $n \in \mathbb{N}$ we construct a collection $\mathscr{G}_{n}$ of open sets in $X$ and an index set $\Delta_{n}$ of $n \times n$ matrices such that for each $\xi \in \Delta_{n}, R_{\xi}, \Omega(\xi), E(\xi), H(\xi)$, and $x_{\xi}$ are given satisfying the following conditions:

(1) Each $\mathscr{G}_{n}$ is locally finite in $X$ such that for each $G \in \mathscr{G}_{n}, \bar{G}$ is disjoint from $A$ or $B$.

(2) For each $\xi \in \Delta_{n},\left\{F\left(\alpha_{1} \cdots \alpha_{k}\right): \alpha_{1}, \ldots, \alpha_{k} \in \Omega(\xi)\right\}, k \in \mathbb{N}$, is a spectral $\sigma$-net of $X_{\xi}$.

(3) For each $\xi=\left(\alpha_{i j}\right)_{1, j \leq n} \in \Delta_{n}$,

(a) $\xi_{-} \in \Delta_{r_{-}-1}, \alpha_{i n} \in \Omega\left(\left(\xi_{-}\right)_{i-1}\right)$ for $1 \leq i \leq n-1$, and $a_{n j} \in \Omega\left(\xi_{-}\right)$ for $1 \leq j \leq n$, where for $n=1, \alpha_{11} \in \Omega\left(\xi_{0}\right)$;

(b) $E(\xi)=\bigcap_{i=1}^{n} p_{\xi_{i-1}}^{-1}\left(F\left(\alpha_{i 1} \cdots \alpha_{i n}\right)\right)$.

(4) $\left\{H(\xi): \xi \in \Delta_{n}\right\}$ is a locally finite collection of open sets of $X$ with $H(\xi) \supset E(\xi)$ for each $\xi \in \Delta_{n}$.

(5) For each $\xi \in \Delta_{n-1}, E(\xi)$ is covered by $\mathscr{G}_{n} \cup\left\{E(\eta): \eta \in \Delta_{n}\right.$ with $\left.\eta_{-}=\xi\right\}$, and $X$ is covered by $\mathscr{G}_{1} \cup\left\{E(\eta): \eta \in \Delta_{1}\right\}$.

(6) For each $\xi \in \Delta_{n}$,

(a) $x_{\xi} \in A \cap E(\xi)$ if $n$ is odd and $x_{\xi} \in B \cap E(\xi)$ if $n$ is even;

(b) $R_{\xi}=R_{\xi_{-}} \cup \operatorname{Supp}\left(x_{\xi}\right)$.

Assume that the above construction has already been performed for no greater than $n$, where, without loss of generality, we may assume that $n$ is odd. Take a $\xi \in \Delta_{n}$. Put

$$
\begin{aligned}
& M_{\xi}=\left\{\eta=\left(\alpha_{i j}\right)_{i, j \leq n+1}: \eta_{-}=\xi, \alpha_{i, n+1} \in \Omega\left(\xi_{i-1}\right)\right. \\
& \text { for } \left.1 \leq i \leq n \text { and } \alpha_{n+1, j} \in \Omega(\xi) \text { for } 1 \leq j \leq n+1\right\} .
\end{aligned}
$$

For each $\eta=\left(\alpha_{i j}\right)_{i, j \leq n+1} \in M_{\xi}$, we define

$$
E(\eta)=\bigcap_{i=1}^{n+1} p_{\xi_{i-1}}^{-1}\left(F\left(\alpha_{i, 1} \cdots \alpha_{i, n+1}\right)\right) .
$$


Moreover, we put

$$
\Delta_{\xi}=\left\{\eta \in M_{\xi}: B \cap E(\eta) \neq \varnothing\right\} .
$$

It is easily seen that $\left\{p_{\xi}(E(\eta)): \eta \in M_{\xi}\right\}$ is a locally finite collection of closed sets of $X_{\xi}$ with $p_{\xi}^{-1} p_{\xi}(E(\eta))=E(\eta)$ for each $\eta \in \Delta_{\xi}$. And so if we define $S(\xi)$ by

$$
S(\xi)=\bigcup\left\{E(\eta): \eta \in M_{\xi} \backslash \Delta_{\xi}\right\},
$$

$P_{\xi}(S(\xi))$ is closed in $X_{\xi}$ with $S(\xi)=p_{\xi}^{-1} p_{\xi}(S(\xi))$. Note that $S(\xi) \subset E(\xi) \cap$ $(X \backslash B)$. It follows from the perfect normality of $X_{\xi}$ that

$$
S(\xi)=\bigcap_{n=1}^{\infty} p_{\xi}^{-1}\left(V_{n}\right) \subset \bigcap_{n=1}^{\infty} \overline{p_{\xi}^{-1}\left(V_{n}\right)} \subset X \backslash B
$$

for some countably many open sets $V_{n}, n \in \mathbb{N}$, in $X_{\xi}$. Since $X$ is countably paracompact, Lemma 1 implies that there exists an open set $G_{\xi}$ in $X_{\xi}$ contained in $H(\xi)$ such that

$$
S(\xi) \subset G_{\xi} \subset \bar{G}_{\xi} \subset X \backslash B
$$

Here, keeping $\xi \in \Delta_{n}$, we let

$$
\mathscr{G}_{n+1}=\left\{G_{\xi}: \xi \in \Delta_{n}\right\} \text { and } \Delta_{n+1}=\bigcup_{\xi \in \Delta_{n}} \Delta_{\xi} .
$$

To define $H(\eta)$ for $\eta \in \Delta_{\xi}$, note, as mentioned above, that $\left\{p_{\xi}(E(\eta)): \eta \in M_{\xi}\right\}$ is a locally finite collection of closed sets of $X_{\xi}$ with $p_{\xi}^{-1} p_{\xi}(E(\eta))=E(\eta) \subset$ $E(\xi)$ for $\eta \in M_{\xi}$. By the paracompactness of $X_{\xi}$, there exists a locally finite collection $\left\{W(\eta): \eta \in M_{\xi}\right\}$ of open sets of $X_{\xi}$ such that $p_{\xi}(E(\eta)) \subset W(\eta)$ and thus $E(\eta) \subset p_{\xi}^{-1}(W(\eta))$. Let $H(\eta)=p_{\xi}^{-1}(W(\eta)) \cap H(\xi)$. It follows from the inductive assumption (4) that $\left\{H(\eta): \eta \in \Delta_{n+1}\right\}$ is locally finite with $H(\eta) \supset E(\eta)$. For each $\eta \in \Delta_{n+1}$, we can choose some $x_{\eta} \in B \cap E(\eta)$. Let $R_{\eta}=R_{\eta_{-}} \cup \operatorname{Supp}\left(x_{\eta}\right)$. Since $X_{\eta}$ is a $\sigma$-space it follows from Lemma 2 that there exists a spectral $\sigma$-net

$$
\left\{F\left(\alpha_{1} \cdots \alpha_{k}\right): \alpha_{1}, \ldots, \alpha_{k} \in \Omega(\eta)\right\}, \quad k \in \mathbb{N},
$$

of $X_{\eta}$ for each $\eta \in \Delta_{n+1}$. Then the conditions (1)-(6) are satisfied for $n+1$. Here we check only (5). Pick any $\xi=\left(\alpha_{i j}\right)_{i, j \leq n} \in \Delta_{n}$. Then

$$
F\left(\alpha_{i 1} \cdots \alpha_{i n}\right)=\bigcup\left\{F\left(\alpha_{i 1} \cdots \alpha_{i, n+1}\right): \alpha_{i, n+1} \in \Omega\left(\xi_{i-1}\right)\right\}
$$

for $i=1, \ldots, n$, and

$$
X=\bigcup\left\{p_{\xi}^{-1}\left(F\left(\alpha_{n+1,1} \cdots \alpha_{n+1, n+1}\right)\right): \alpha_{n+1, j} \in \Omega(\xi) \text { for } j=1, \ldots, n+1\right\} .
$$

It follows that

$$
E(\xi)=\bigcup\left\{E(\eta): \eta \in M_{\xi}\right\} \subset \bigcup \mathscr{G}_{n+1} \cup\left(\bigcup\left\{E(\eta): \eta \in \Delta_{\xi}\right\}\right) .
$$

We now set $\mathscr{G}=\bigcup_{n=1}^{\infty} \mathscr{G}_{n}$. By (1), $\mathscr{G}$ is a $\sigma$-locally finite collection of open sets of $X$ such that for $G \in \mathscr{G}, \bar{G}$ misses $A$ or $B$. To complete the proof, it suffices to show that $\mathscr{G}$ covers $X$. Assume the contrary and pick some $x \in$ $X \backslash \bigcup \mathscr{G}$. By (2) and (5), we can inductively choose a sequence $\left\{\alpha_{i j}: i, j \geq 1\right\}$ such that for each $n \geq 1, \xi^{(n)}=\left(\alpha_{i j}\right)_{i, j \leq n} \in \Delta_{n}$ and $\left\{F\left(\alpha_{n 1} \cdots \alpha_{n k}\right): k \in \mathbb{N}\right\}$ 
is a local $\sigma$-net of $X_{\xi^{(n-1)}}$ at point $p_{\xi^{(n-1)}}(x)$, where $\alpha_{n k} \in \Omega\left(\xi^{(n-1)}\right)$ and $\xi^{(0)}=$ $\xi_{0}$. Now fix $m \geq 1$. If $n>m$, then

$$
x_{\xi(n)} \in E\left(\xi^{(n)}\right) \subset p_{\xi(m)}^{-1}\left(F\left(\alpha_{m+1,1} \cdots \alpha_{m+1, n}\right)\right) .
$$

We thus have $p_{\xi(m)}\left(x_{\xi(n)}\right) \in F\left(\alpha_{m+1,1} \cdots \alpha_{m+1, n}\right)$ for each $n>m$. Since $\left\{F\left(\alpha_{m+1,1} \cdots \alpha_{m+1, k}\right): k \in \mathbb{N}\right\}$ is a local $\sigma$-net of $X_{\xi(m)}$ at point $p_{\xi(m)}(x)$, the sequence $\left\{p_{\xi(m)}\left(x_{\xi(n)}\right)\right\}_{n>m}$ converges to $p_{\xi(m)}(x)$. Define a point $y=\left(y_{\lambda}\right)_{\lambda \in \Lambda}$ in $X$ by letting $y_{\lambda}=x_{\lambda}$ if $\lambda \in \bigcup_{n=1}^{\infty} R_{\xi(n)}$ and $y_{\lambda}=s_{\lambda}$ otherwise. Then one can prove that the sequence $\left\{x_{\xi_{(n)}}\right\}_{n \in \mathbb{N}}$ converges to $y$, and thus $y \in A \cap B$. This is a contradiction. The proof of Theorem 1 is complete.

Question 2 is still open. By Theorem 1 we now have

Corollary 1. A $\Sigma$-product of Lašnev $\left(M_{1^{-}}\right)$spaces is normal if and only if it is countably paracompact.

By Theorem 1 and [20, Corollary 1] we also have

Corollary 2. The following are equivalent for a $\Sigma$-product $X$ of paracompact $\sigma$-spaces.

(1) $X$ is collectionwise normal.

(2) $X$ is shrinking.

(3) $X$ is normal.

(4) $X$ is countably paracompact.

It is not possible to replace $\sigma$-spaces by $\Sigma$-spaces in Theorem 1 . Since, as pointed out in the abstract, $\Sigma$-products of compact spaces always are countably paracompact but not necessarily normal [4].

\section{Proof of Theorem 2}

A space is said to be semistratifiable [3] if there exists a function $g$ of $X \times \mathbb{N}$ into the topology of $X$ satisfying

(i) $\bigcap_{n \in \mathbb{N}} g(x, n)=\{x\}$ for each $x \in X$;

(ii) if $\left\{x_{n}\right\}$ is a sequence of points in $X$ with $x \in \bigcap_{n \in \mathbb{N}} g\left(x_{n}, n\right)$ for some $x \in X$, then $\left\{x_{n}\right\}$ converges to $x$.

A space $X$ is said to be shrinking if for every open cover $\left\{G_{\gamma}: \gamma \in \Gamma\right\}$ of $X$ there exists a closed cover $\left\{F_{\gamma}: \gamma \in \Gamma\right\}$ of $X$ such that $F_{\gamma} \subset G_{\gamma}$ for each $\gamma \in \Gamma$. If the closed cover can be weakly chosen as a closed cover $\mathscr{F}=\left\{F_{\gamma, n}: \gamma \in \Gamma\right.$ and $n \in \mathbb{N}\}$ with $F_{\gamma, n} \subset G_{\gamma}$ for each $\gamma \in \Gamma$ and $n \in \mathbb{N}$, then the space is said to be subshrinking. Such a cover $\mathscr{F}$ is called a subshrinking of $\left\{G_{\gamma}: \gamma \in \Gamma\right\}$.

It follows from Bešlagic [1] that a space is shrinking if and only if it is normal and subshrinking. Note that subparacompact spaces are subshrinking.

Proof of Theorem 2. Let $X$ be a semistratifiable space and $\mathscr{G}=\left\{G_{\gamma}: \gamma \in \Gamma\right\}$ an open cover of $X \times \kappa$. We shall find a subshrinking for $\mathscr{G}$.

For a set $F \subset X$, set

$\mathscr{W}(F)=\left\{W: W\right.$ is open in $\kappa$ such that $F \times W \subset G_{\gamma}$ for some $\left.\gamma \in \Gamma\right\}$

and

$$
\mathscr{V}=\{V: V \text { is open in } X \text { such that } \kappa=\bigcup \mathscr{W}(V)\}
$$


Now for each $n \in \mathbb{N}$ we construct inductively two $\sigma$-locally finite collections $\mathscr{G}_{n}$ and $\mathscr{F}_{n}$ of closed sets of $X$ satisfying the following conditions (1)-(3):

(1) $\mathscr{F}_{n+1}$ can be expressed as $\mathscr{F}_{n+1}=\bigcup\left\{\mathscr{F}_{F} \in \mathscr{F}_{n}\right\}$.

(2) For each $C \in \mathscr{C}_{n}, \kappa=\bigcup \mathscr{W}(C)$.

(3) For each $F \in \mathscr{F}_{n}$,

(a) $F \subset g\left(x_{F}, n\right)$ for some $x_{F} \in X \backslash \bigcup \mathscr{V}$;

(b) $F$ is covered by $\mathscr{C}_{n+1} \cup \mathscr{F}_{F}$; and $X$ is covered by $\mathscr{C}_{1} \cup \mathscr{F}_{1}$.

Assume $n \in \mathbb{N}$, and $\mathscr{C}_{i}$ and $\mathscr{F}_{i}$ for $i \leq n$ have already been defined satisfying the conditions. Take an $F \in \mathscr{F}_{n}$ and fix it. Put

$$
\mathscr{W}=\{F \cap V: V \in \mathscr{V}\} \cup\{F \cap g(x, n+1): x \in F \backslash \bigcup \mathscr{V}\}
$$

By the subparacompactness of $X$, there exists a $\sigma$-locally finite closed cover $\mathscr{F}$ of $F$ refining $\mathscr{W}$. Let $\mathscr{C}_{F}=\{F \in \mathscr{F}: F \subset V$ for some $V \in \mathscr{V}\}$ and $\mathscr{F}_{F}=\mathscr{F} \backslash \mathscr{C}_{F}$. Here running $F \in \mathscr{F}_{n}$ we put

$$
\mathscr{C}_{n+1}=\bigcup\left\{\mathscr{C}_{F}: F \in \mathscr{F}_{n}\right\} \text { and } \mathscr{F}_{n+1}=\bigcup\left\{\mathscr{F}_{F}: F \in \mathscr{F}_{n}\right\} .
$$

Then both $\mathscr{C}_{n+1}$ and $\mathscr{F}_{n+1}$ are locally finite satisfying conditions (1)-(3).

Claim. $\mathscr{C}=\bigcup_{n=1}^{\infty} \mathscr{C}$ covers $X$.

Assume the contrary and pick some $x \in X \backslash \bigcup \mathscr{C}$. Then one can easily find a sequence $\left\{x_{n}\right\}_{n \in \mathbb{N}}$ in $X \backslash \bigcup \mathscr{V}$ such that $x \in g\left(x_{n}, n\right)$ for each $n \in \mathbb{N}$. It follows from the definition of semistratifiable spaces above Definition 3 that the sequence $\left\{x_{n}\right\}_{n \in \mathbb{N}}$ converges to $x$. Since $\chi(X)<\kappa$, we may take a $\lambda<\kappa$ and find a nbd base $\left\{V\left(x_{n}, \alpha\right): \alpha<\lambda\right\}$ for $x_{n}, n \in \mathbb{N}$. By the definition of $x_{n}$, for each $n \in \mathbb{N}$ there exists a point

$$
\xi(n, \alpha) \in \kappa \backslash \bigcup \mathscr{W}\left(V\left(x_{n}, \alpha\right)\right)
$$

for each $\alpha<\lambda$. Let $\xi(n)$ be a cluster point of the net $\{\xi(n, \alpha): \alpha<\kappa\}, n \in \mathbb{N}$, and let $\xi$ be a cluster point of the sequence $\{\xi(n)\}_{n \in \mathbb{N}}$. Then $(x, \xi) \in G_{\gamma}$ for some $\gamma \in \Gamma$. It is not hard to find an $n$, an $\alpha<\lambda$, and a nbd of $O_{\xi}$ of $\xi$ such that

$$
V\left(x_{n}, \alpha\right) \times O_{\xi} \subset G_{\gamma} .
$$

It follows that $\xi(n, \alpha) \in \bigcup \mathscr{W}\left(V\left(x_{n}, \alpha\right)\right)$.

Now decompose $\mathscr{C}$ as $\mathscr{C}=\bigcup_{n=1}^{\infty} \mathscr{C}_{n}^{\prime}$ so that $\mathscr{C}_{n}^{\prime}$ is locally finite. Pick a $C \in \mathscr{C}$ and fix it. For each $\alpha \in \kappa$, there exists an $f(\alpha)<\alpha$ such that $C \times(f(\alpha), \alpha] \subset G_{\gamma}$ for some $\gamma \in \Gamma$; let $\gamma(\alpha, C)$ denote this $\gamma$. By the Pressing Down Lemma, there exists a $\beta \in \kappa$ and a stationary set $S \subset \kappa$ such that $f(\alpha)=\beta$ for all $\alpha \in S$. Therefore we have $C \times(\beta, \alpha] \subset G_{\gamma(\alpha, C)}$ for all $\alpha \in S$. Without loss of generality we can assume that either all $\gamma(\alpha, C), \alpha \in S$, are the same or different. If all $\gamma(\alpha, C), \alpha \in S$, are the same, we may put $\gamma(C)=\gamma(\alpha, C)$ for all $\alpha \in S$. We let $\beta_{C}$ denote the chosen $\beta$ and index the chosen stationary set $S$ as $S=\{\alpha(C, \mu): \mu \in \kappa\}$. Here keeping $C \in \mathscr{C}$, decompose $\mathscr{C}_{n}^{\prime}$ for each $n \in \mathbb{N}$ as

$$
\mathscr{C}_{n}^{\prime}(1)=\cdot\left\{C \in \mathscr{C}_{n}^{\prime}: \text { all } \gamma(\alpha(C, \mu), C), \mu<\kappa, \text { are the same }\right\}
$$


and $\mathscr{C}_{n}^{\prime}(2)=\mathscr{C}_{n}^{\prime} \backslash \mathscr{C}_{n}^{\prime}(1)$. We now put

$$
\begin{aligned}
& H_{n \gamma}=\left(\bigcup\left\{C \times\left(\beta_{C}, \kappa\right): C \in \mathscr{C}_{n}^{\prime}(1) \text { with } \gamma(C)=\gamma\right\}\right) \\
& \cup\left(\bigcup \left\{C \times\left(\beta_{C}, \alpha(C, \mu)\right]: C \in \mathscr{C}_{n}^{\prime}(2) \text { and } \mu<\kappa\right.\right. \\
&\text { with } \gamma(\alpha(C, \mu), C)=\gamma\})
\end{aligned}
$$

for each $\gamma \in \Gamma$ and $n \geq 1$. Then $H_{n \gamma}$ is a closed set in $X \times \kappa$ with $H_{n \gamma} \subset G_{\gamma}$ for each $\gamma \in \Gamma$ and $n \geq 1$.

Moreover, for each $C \in \mathscr{C}$, since the subspace $C \times\left[0, \beta_{C}\right]$ is subparacompact, there exists a closed cover $\left\{Z_{n, C, \gamma}: n \in \mathbb{N}\right.$ and $\left.\gamma \in \Gamma\right\}$ of it such that $Z_{n, C, \gamma} \subset G_{\gamma}$ for each $n \in \mathbb{N}$ and $\gamma \in \Gamma$. Let us set

$$
H_{n, m, \gamma}=\bigcup\left\{Z_{n, C, \gamma}: C \in \mathscr{C}_{m}^{\prime}\right\}
$$

for each $n, m \in \mathbb{N}$ and $\gamma \in \Gamma$. It is easy to see that $H_{n, m, \gamma}$ is closed with $H_{n, m, \gamma} \subset G_{\gamma}$ for each $n, m \in \mathbb{N}$ and $\gamma \in \Gamma$. So we find a subshrinking

$$
\left\{H_{n, m, \gamma}: n, m \in \mathbb{N} \text { and } \gamma \in \Gamma\right\} \cup\left\{H_{n \gamma}: n \in \mathbb{N} \text { and } \gamma \in \Gamma\right\}
$$

for the open cover $\mathscr{G}$ which completes the proof.

Notice that for any subparacompact space $X$ Yajima [21] gives a sufficient condition for $X \times \kappa$ to be subshrinking.

\section{ACKNOWLEDGMENT}

The author would like to thank Professor T. Hoshina for his useful help with this paper.

\section{REFERENCES}

1. A. Bešlagić, Normality in products, Topology Appl. 22 (1986), 71-88.

2. H. H. Corson, Normality in subsets of product spaces, Amer. J. Math. 81 (1959), 785-796.

3. G. D. Creede, Concerning semi-stratifiable spaces, Pacific J. Math. 32 (1970), 47-54.

4. S. P. Gul'ko, On the properties of $\Sigma$-products, Soviet Math. Dokl. 18 (1977), 1438-1442.

5. T. Hoshina, Products of normal spaces with Lasnev spaces, Fund. Math. 124 (1984), 143153.

6. __ Shrinking and normal products, Questions Answers Gen. Topology 2 (1984), 83-91.

7. __ Normality of product spaces. II, Topics in General Topology (K. Morita and J. Nagata, eds.), North-Holland, Amsterdam, 1989.

8. A. P. Kombarov, On tightness and normality of $\Sigma$-products, Soviet Math. Dokl. 19 (1978), 403-407.

9. K. Morita, Note on products of normal spaces with metric spaces, unpublished.

10. K. Nagami, $\sigma$-spaces and product spaces, Math. Ann. 181 (1969), 109-118.

11. Countable paracompactness of inverse limits and products, Fund. Math. 73 (1972), 261-270.

12. __ $\Sigma$-spaces, Fund. Math. 65 (1969), 169-192.

13. J. Nagata, Modern general topology, North-Holland, Amsterdam, 1985.

14. T. C. Przymusinski, Products of normal spaces, Handbook of Set Theoretic Topology (K. Kunen and J. Vaughan, eds.), North-Holland, Amsterdam, 1984. 
15. M. E. Rudin, Book Review, Bull. Amer. Math. Soc. 84 (1978), 271-272.

16. _ The shrinking property, Canad. Math. Bull. 26 (1983), 385-388.

17. M. E. Rudin and M. Starbird, Products with a metric factor, Gen. Topology Appl. 5 (1975), 235-248.

18. Y. Yajima, On $\Sigma$-products of $\Sigma$-spaces, Fund. Math. 123 (1984), 29-37.

19. _ On $\Sigma$-products of semi-stratifiable spaces, Topology Appl. 25 (1987), 1-11.

20. _ The shrinking property of $\Sigma$-products, Tsukuba J. Math. 13 (1989), 83-98.

21. _ Subnormality of $X \times \kappa$ and $\Sigma$-products, Topology Appl. (to appear).

22. P. Zenor, Countable paracompactness in product spaces, Proc. Amer. Math. Soc. 30 (1971), 199-201.

Institute of Mathematics, University of Tsukuba, Tsukuba-shi 305, Japan 